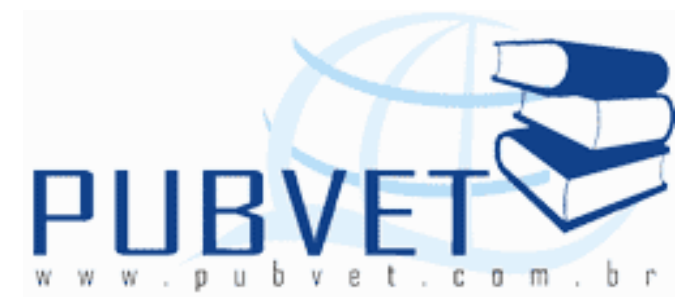

PUBVET, Publicações em Medicina Veterinária e Zootecnia.

\title{
O ciclo do nitrogênio na criação de frangos de corte e suas perdas na forma de amônia volátil: uma revisão
}

Luciano Barreto Mendes $^{(1)}$, Ilda de Fátima Ferreira Tinôco ${ }^{(2)}$, Cecília de Fátima Souza ${ }^{(2)}$, Jairo Alexander Osorio Saraz ${ }^{(3)}$

${ }^{(1)}$ Doutorando em Construções Rurais e Ambiência, Departamento de Engenharia Agrícola, Universidade Federal de Viçosa, Viçosa. E-mail: mrmendes2010@gmail.com;

${ }^{(2)}$ Professora Associada, Departamento de Engenharia Agrícola, Universidade Federal de Viçosa, Viçosa. E-mail: iftinoco@ufv.br; cfsouza@ufv.br;

${ }^{(3)}$ Professor Associado, Departamento de Engenharia Agrícola, Universidad Nacional de Colombia, Medellín, Colombia. E-mail: aosorio@unal.edu.co.

\section{Resumo}

A produção avícola brasileira tem alcançado índices sem precedentes nos últimos anos, especialmente no que tange à criação de frangos de corte. Tal produtividade tem aumentado graças ao incremento progressivo de tecnologia neste setor da produção animal brasileira, especialmente na melhoria da ambiência e também aperfeiçoamento nas dietas oferecidas às aves e melhoramentos genético das mesmas. Juntamente com alta produtividade de frangos de corte, vem a produção também exarcebada de resíduos, e em especial da cama usada, a qual possui altos teores de nutrientes, dentre os quais está o nitrogênio $(\mathrm{N})$, o que torna a cama avícola um material biológico 
com alto potencial para ser utilizado como fertilizante. Uma das formas de empobrecimento da qualidade da cama é através da volatilização do $\mathrm{N}$ na forma de amônia $\left(\mathrm{NH}_{3}\right)$. A ciclagem do $\mathrm{N}$ como um todo em galpões avícolas de frangos de corte tem sido pouco tratada na literatura brasileira até então, e por esta razão esta revisão bibliográfica foi elaborada, no intuito de destrinchar todas as possíveis fontes e sumidouros, bem como os mecanismos de transformação do $\mathrm{N}$ no galpão. Um foco especial foi dado aos processos de formação e modelos de volatilização da $\mathrm{NH}_{3}$. Como principal conclusão, observou-se que o aumento do índice de conversão alimentar com a idade é um dos fatores que mais contribuem para o não-aproveitamento do $\mathrm{N}$ oferecido aos frangos, implicando num maior teor deste nutriente na cama com o aumento da idade e aumento do potencial de sua perda através da $\mathrm{NH}_{3}$ volatilzável.

Termos para indexação: Poluição do ar, nitrogênio, frangos de corte, galpões avícolas

\title{
The nitrogen cycle and loss within a broiler chicken barn: a review
}

\begin{abstract}
Brazilian poultry production has reached unprecedented levels in recent years, especially regarding to the broiler chickens production activity. This increasing productivity is due to progressive implementation of technology in this sector of the animal production in Brazil, which is being mainly focused on improving the animal environment and also, the diets offered to the birds combined with genetic advances. One consequence of high productivity of broiler chickens is the exacerbated production of waste, especially used litter, which has high levels of nutrients, among which is the nitrogen $(\mathrm{N})$, that makes the poultry litter a material with high biological potential to be used as fertilizer. One way of depleting the quality of the litter is through volatilization of $N$ as gaseous ammonia $\left(\mathrm{NH}_{3}\right)$. The cycling of $\mathrm{N}$ for a whole broiler barn has been poorly treated in the current Brazilian scientific literature, which is the reason why
\end{abstract}


this literature review was developed. The aim was to depict all possible sources and sinks and the mechanisms transformation of $\mathrm{N}$ inside the barn. Special focus was given to the processes of formation and models of $\mathrm{NH}_{3}$ volatilization. As main conclusion, we observed that the increase in feed conversion ratio with age is one of the factors that contributed to non-utilization of $\mathrm{N}$ offered to broilers, implying in a higher concentration of this nutrient in the litter with increasing age and consequently potential of its loss through volatilized $\mathrm{NH}_{3}$.

Indexation terms: Air pollution, nitrogen, broiler chickens, poultry barns

\section{Introdução}

Quatro quintos da atmosfera terrestre são constituídos por nitrogênio molecular $\left(\mathrm{N}_{2}\right)$, em que esta é a forma mais abundante deste elemento. Juntamente com o carbono (C), o nitrogênio (N) constitui um dos mais complexos e cruciais elementos essenciais para a existência de vida no planeta Terra (Krupa, 2003).

Já na década de 1650 o alquimista alemão Johannes Rudolph Glauber descreveu o nitrium (nome primitivo dado ao $\mathrm{N}$ pelos alquimistas) como sendo "uma ave sem asas que voa dia e noite sem descansar; que penetra todos os elementos (água, fogo, terra e ar) e carrega consigo o espírito da vida; ele muda de forma; penetra na constituição corporal dos animais através do alimento, sendo em seguida excretado. O nitrium retorna, então, ao solo, do qual novamente será vertido ao ar em forma de vapores, e mais uma vez por entre os elementos da natureza" (Follet e Hatfield, 2009).

O que o alquimista alemão descrevia pela primeira vez na história de forma sucinta e poética, era o ciclo do $\mathrm{N}$, que em suas formas orgânica (alimento que penetra na constituição dos animais) e inorgânica (gases exalados pelo solo) permeiam por praticamente todos os recintos da natureza.

Entre 1850 e 1980, muitos cientistas mantiveram-se focados em revelar as intrínsecas relações biológicas e físico-químicas na natureza que envolvem o N. Hoje, são conhecidas praticamente todas as "idas e vindas" deste elemento 
e as rotas que ele toma enquanto se desloca em taxas que podem variar de milissegundos a séculos, nos compartimentos da natureza (atmosfera, solo, água e matéria viva), bem como sua interação com outros elementos (Follet e Hatfield, 2009).

$\mathrm{O}$ ciclo do $\mathrm{N}$ é classificado como um ciclo biogeoquímico, uma vez que tanto processos químicos quanto biológicos e geológicos provêm importantes inputs para o ciclo global. A ciclagem do $\mathrm{N}$ envolve processos cinéticos, os quais podem, por sua vez, envolver o consumo, absorção, armazenamento e transporte de energia. A principal fonte de energia que alimenta as reações que fazem o ciclo do $\mathrm{N}$ funcionar é o Sol. A Terra recebe do Sol uma média de 100 a $300 \mathrm{~W} \mathrm{~m}^{-2} \mathrm{dia}^{-1}$, a depender da latitude. Parte desta energia é capturada através da fotossíntese e posteriormente utilizada para produzir moléculas com alto teor energético, como o gás oxigênio $\left(\mathrm{O}_{2}\right)$ (Erisman et al., 2007).

Nitrificação, desnitrificação, formação de óxido nítrico, lixiviação de nitratos e volatilização da amônia $\left(\mathrm{NH}_{3}\right)$ são possíveis caminhos de transformação que podem ser sofridas pelas moléculas que contém N. No entanto, vários problemas ecológicos podem ocorrer quando o $\mathrm{N}$ é separado de seu parceiro mais comum, o carbono (Asner et al., 1997; Follet e Hatfield, 2009). Dada a demanda mundial por alimentos, a habilidade excepcional dos grãos de ciclo anual e semi-anial em produzir o alimento necessário para a humanidade, e ração animal, aliados com o custo relativamente baixo dos fertilizantes nitrogenados, problemas ambientais surgem graças ao excesso do $\mathrm{N}$ em algumas de suas formas, causando estagnação de certos compostos nitrogenados ou acelerando certos processos relacionados ao ciclo (Fallet e Hatfield, 2009). Por esta razão, a observação detalhada do caminho percorrido pelo $\mathrm{N}$ em sistemas naturais ou semi-naturais pode ser de grande valia para se compreender onde o $\mathrm{N}$ está sendo utilizado de forma eficiente, acumulado ou perdido através de outras formas e para outros sistemas.

As perdas de $\mathrm{N}$ do sistema de interesse (por exemplo, um galpão que aloja animais, ou uma área cultivada) para outros sistemas vizinhos (ecossistema das redondezas) geralmente constituem um aspecto negativo por 
empobrecer o sistema de interesse (esterco ou cama dos animais confinados, ou o poder fertilizante de um fertilizante), ao passo em que seu excesso em ecossistemas vizinhos sensíveis às formas de $\mathrm{N}$ perdidas, poderão causar desequilíbrio ecológico (Sutton et al., 2008).

Os sistemas de produção animal, sobretudo em confinamento, constituem importantes exemplos nos quais $\mathrm{N}$ entra em altas quantidades, através de rações ricas em aminoácidos no intuito de melhorar o desempenho produtivo dos animais (Yang et al., 2000). Dentre os sistemas de confinamento animal, a indústria avícola tem se destacado a nível mundial por vários motivos, os quais incluem: qualidade dos produtos gerados (ovos e carne, que são fontes de proteína de ótima qualidade para a dieta humana e de baixo custo), ciclos curtos (no caso dos frangos de corte) e nível de tecnologia relativamente alto, o que torna a produção mais homogênea e eficiente.

Vários artigos têm reportado as perdas de $\mathrm{N}$ a partir dos resíduos avícolas (esterco e cama). Com base no teor total de $\mathrm{N}$ medido no esterco, ovos e carcaças num sistema de alojamento de poedeiras, $40 \%$ do $\mathrm{N}$ que entrou na forma de ração foi perdido para a atmosfera na forma de $\mathrm{NH}_{3}$ (Patterson e Lorenz, 1996; Yang et al., 2000). De fato, Hartung (1990), citado por Yang et al. (2000) reportou que $37 \%$ de todo o $\mathrm{N}$ perdido por sistemas de confinamento de animais é transportado para a atmosfera na forma de $\mathrm{NH}_{3}$. Em geral, as perdas de $\mathrm{N}$ na forma gasosa são consideradas em termos de $\mathrm{N}$ amoniacal $\left(\mathrm{N}-\mathrm{NH}_{3}\right)$ através do ar que passa pela estrutura de alojamento (Yang et al., 2000).

Para o cálculo matemático da perda de $\mathrm{N}$ de um sistema, o conceito de balanço de $\mathrm{N}$ (em base mássica) tem sido aplicado em vários estudos (Liang, 2004; Keener and Zhao, 2008; Yang et al, 2000). De acordo com a teoria do balanço de massa, a diferença entre os fluxos de entrada e saída de $\mathrm{N}$ é igual à taxa de emissão (perda) de $\mathrm{N}$ (ou $\mathrm{NH}_{3}$ ). Assim, a perda total de $\mathrm{N}$ para um sistema de produção avícola pode ser calculado pela Equação 1. 
MENDES, L.B. et al. O ciclo do nitrogênio na criação de frangos de corte e suas perdas na forma de amônia volátil: uma revisão. PUBVET, Londrina, V. 6, N. 20, Ed. 207, Art. 1383, 2012.

$N_{\text {perdido }}=N_{\text {ração }}-\left(N_{\text {Esterco ou cama }}+N_{\text {ovos }}+N_{\text {Aves vivas }}+N_{\text {Aves mortas }}\right)$

Para o caso específico de um sistema de criação de frangos de corte, o ciclo do $\mathrm{N}$ compreenderá a ração, como fonte principal de $\mathrm{N}$, enquanto que a cama usada, animais mortos, e as emissões gasosas de compostos nitrogenados são considerados como formas de escape de $\mathrm{N}$ do sistema. Outras formas de escape como a lixiviação através do solo também ocorrem, porém em menor magnitude uma vez que as instalações de confinamento geralmente possuem pisos concretados (Tinôco, 2004). A seguir será feita uma descrição das fontes e sumidouros de $\mathrm{N}$ mais comuns num sistema de criação de frangos de corte.

\section{Principais fontes e sumidouros de $\mathbf{N}$ num galpão avícola}

\subsection{Ração}

O desempenho de frangos de corte é função direta da ração oferecida aos mesmos. Recomendações de formulação da ração são fornecidas pelas empresas que testam cada nova linhagem de frango que sai no mercado, de modo que uma massa corporal específica seja alcançada em cada idade, no intuito de alcançar a eficiência máxima. Por exemplo, na Figura 1 pode-se observar o peso corporal esperado para frangos de corte da linhagem Cobb500® em função da idade (0 a 45 d). Para atingir tais objetivos, rações com teores elevados de energia (2988 a 3176 kcal kg-1) e proteínas (17 a $21 \%$ ) são administradas para as aves. Observa-se ainda na Figura 1 que o consumo diário de ração aumenta com a idade, para suprir as necessidades crescentes de nutrientes dos animais, uma vez que na medida em que as aves envelhecem, uma quantidade maior de energia é necessária para suprir suas necessidades diárias (Tabela 1) e continuar ganhando peso, o que implica num maior índice de conversão alimentar (CA). 


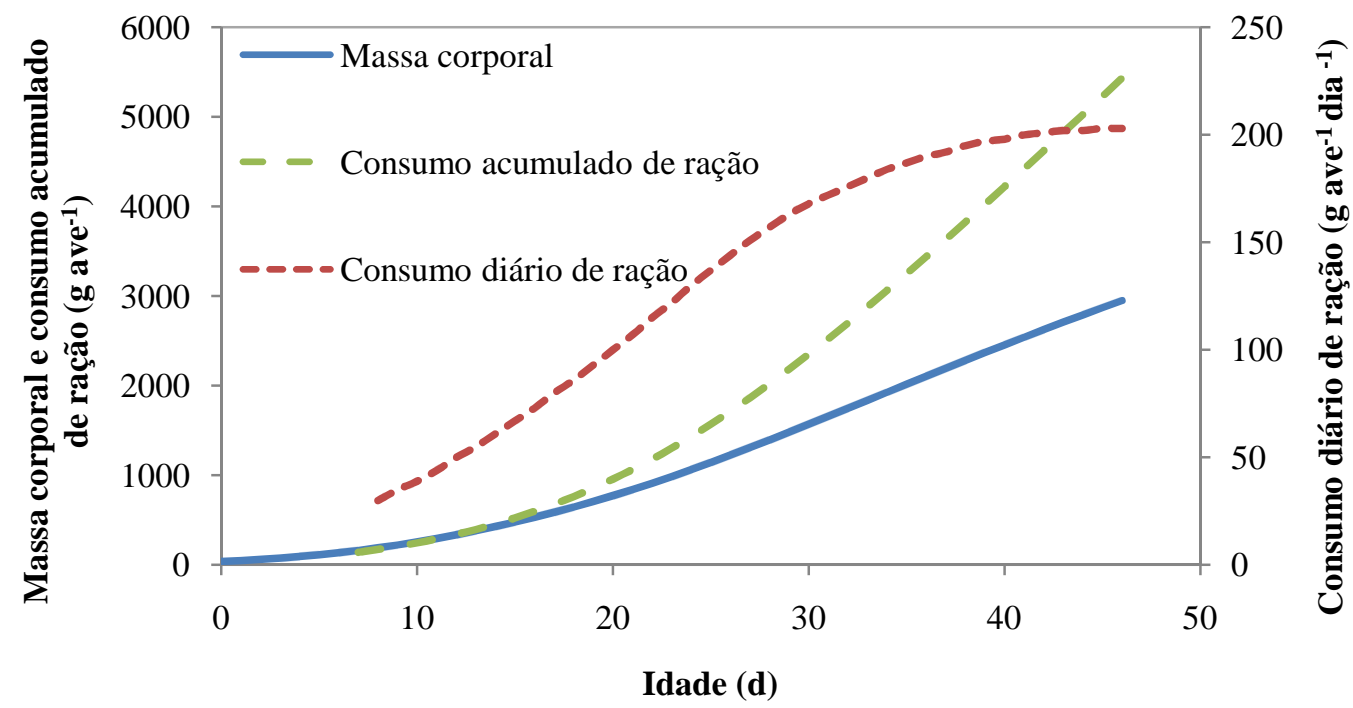

Figura 1. Massa corporal ( $\left(\mathrm{ave}^{-1}\right)$ e consumos acumulado e diário de ração ( $\mathrm{g}$ $\mathrm{ave}^{-1}$ e g ave $\mathrm{dia}^{-1}$, respectivamente) em função da idade (d) para um lote misto ( $50 \%$ machos e $50 \%$ fêmeas) de frangos de corte da linhagem Cobb500® (Adaptado de Cobb-Vantress, 2009).

$\mathrm{Na}$ Tabela 1 podem-se observar os valores nutricionais recomendados para frangos de corte da linhagem Cobb $500 \AA$. Nota-se que a composição recomendada varia de acordo com a idade, havendo quatro principais tipos de ração: a ração inícial, a de crescimento e a ração para os estágios finais 1 e 2 . Da observação dos dados da Tabela 1 , pode-se notar ainda que o teor de proteína bruta na ração é diminuído com a idade, enquanto que o teor energético da mesma aumenta.

O aumento do teor energético da ração sugerido na Tabela 1 pode ser explicado pelo fato de que com o aumento da idade, as aves tendem a apresentar um maior índice de conversão alimentar, implicando que a ingestão de uma quantidade maior de alimento é necessária para ganho de massa corpórea, em relação a aves mais jovens. O aumento do índice de conversão alimentar (CA) acumulado com a idade para a linhagem Cobb $500 \AA$, pode ser observado na Figura 3, onde se nota que para pintos com idade menor que 12 d, criados em condições ambientais adequadas e recebendo ração balanceada, 
MENDES, L.B. et al. O ciclo do nitrogênio na criação de frangos de corte e suas perdas na forma de amônia volátil: uma revisão. PUBVET, Londrina, V. 6, N. 20, Ed. 207, Art. 1383, 2012.

o CA é < 1. Por outro lado, para aves adultas a CA pode ultrapassar 1,8 a partir do $18^{\circ} \mathrm{d}$.

Tabela 1. Recomendações de formulação da ração para frangos de corte da linhagem Cobb500®.

\begin{tabular}{ccccc}
\hline Período & Início & Crescimento & Final 1 & Final 2 \\
\hline $\begin{array}{c}\text { Quantidade de ração (g ave }{ }^{-1} \text { ) } \\
\text { Período de administração da }\end{array}$ & 250 & 1000 & - & \\
ração (d) & $0-10$ & $11-22$ & $21-42$ & $42+$ \\
Proteína bruta (\%) & 21 & 19 & 18 & 17 \\
Energia metabolizável & 2988 & 3083 & 3176 & 3176 \\
(kcal kg-1) & & & & \\
Lisina (\%) & 1,20 & 1,10 & 1,05 & 1,00 \\
Lisina digestível (\%) & 1,08 & 0,99 & 0,95 & 0,90 \\
Metionina (\%) & 0,46 & 0,44 & 0,43 & 0,41 \\
Metionina digestível (\%) & 0,41 & 0,40 & 0,39 & 0,37 \\
Met. + cis. (\%) & 0,89 & 0,84 & 0,82 & 0,78 \\
Met. + cis. digestível (\%) & 0,80 & 0,75 & 0,74 & 0,70 \\
Triptófano (\%) & 0,20 & 0,19 & 0,19 & 0,18 \\
Treonina (\%) & 0,79 & 0,74 & 0,72 & 0,69 \\
Arginina (\%) & 1,26 & 1,17 & 1,13 & 1,08 \\
Cálcio (\%) & 1,00 & 0,96 & 0,90 & 0,85 \\
Fósforo disponível (\%) & 0,50 & 0,48 & 0,45 & 0,42 \\
Sódio (\%) & 0,22 & 0,19 & 0,19 & 0,18 \\
Cloro (\%) & 0,20 & 0,20 & 0,20 & 0,20 \\
Proporção cal:proteína & 142 & 162 & 176 & 187 \\
\hline \hline
\end{tabular}

(Fonte: Cobb-Vantress, 2009) 


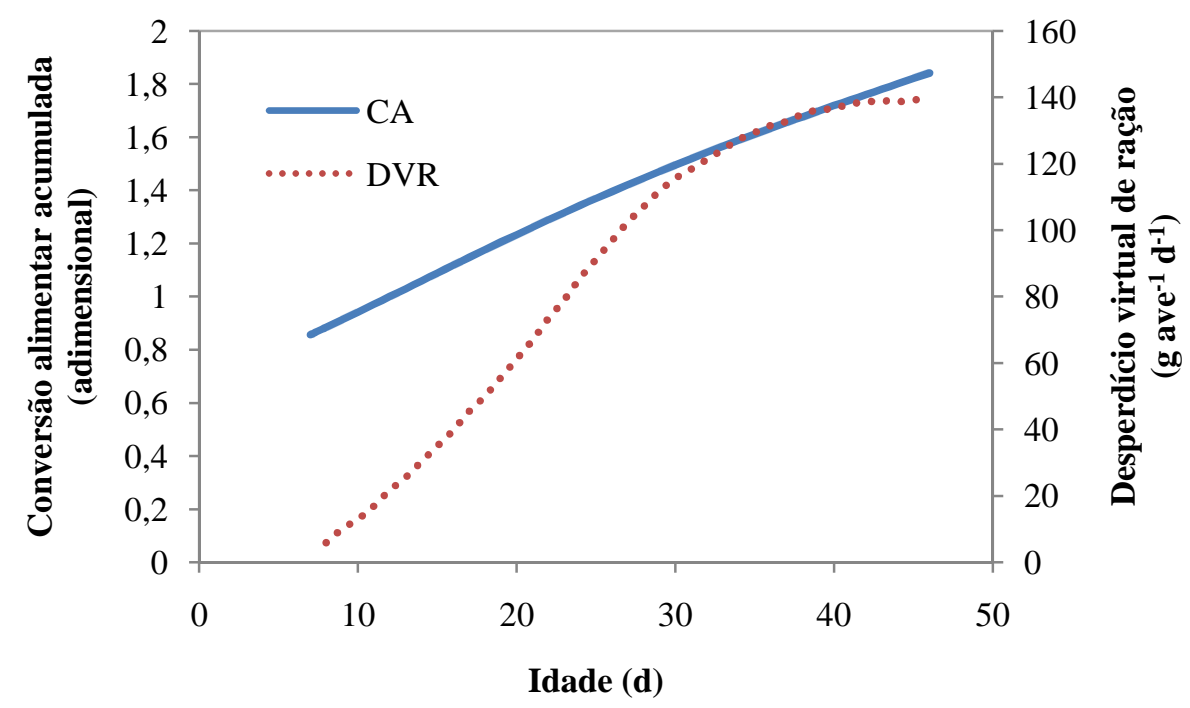

Figura 2. Conversão alimentar (CA) e desperdício virtual de ração (DVR, g ave ${ }^{1} \mathrm{~d}^{-1}$, termo criado pelos autores) para frangos de corte da linhagem Cobb500® como uma função da idade, para aves criadas nas condições ambientais ideais e recebendo ração balanceada (Adaptado de Cobb-Vantress, 2009).

Os valores de CA fornecidos pela Cobb-Vantress (2009) foram combinados com os dados de consumo diário de ração e ganho diário de massa corporal para se obter o desperdício virtual de ração (DVR, g ave $e^{-1} d^{-1}$, termo criado pelos autores). A curva da evolução do DVR com a idade da ave é apresentada na Figura 3, onde se observa que a mesma pode variar de 0 a $140 \mathrm{~g} \mathrm{ave}^{-1} \mathrm{dia}^{-1}$. O DVR apresentado aqui serve para dar uma idéia do total da ração ingerida que não foi absorvida pelo trato digestivo das aves, tendo sido excretada pelas mesmas a posteriori, e depositada na cama, estando susceptível á degradação microbiológica e subsequente volatilização dos produtos da decomposição. 


\subsection{Cama aviária}

Em um galpão avícola, dá-se o nome de cama a qualquer substrato orgânico, o qual paulatinamente mistura-se com os excrementos das aves, penas e restos de ração. A cama tem por função impedir o contato direto do animal com o piso da instalação, melhorando as condições de conforto, higiene e reduzindo as trocas de calor entre o animal e o solo por possuir boa resistência térmica (Tinôco, 2004).

A cama é geralmente reutilizada por até 3 lotes, havendo casos em que o uso é feito por um número muito maior de ciclos. Os substratos utilizados hoje em dia para produzir cama são subprodutos industriais ou restos de culturas agrícolas, como: maravalha, resíduos de beneficiamento industrial da madeira, sabugo de milho triturado, casca de arroz, palhadas de culturas em geral, fenos de gramíneas e cascas de amendoim (Tinôco, 2002). Desta forma, existem vários materiais que podem servir como cama, sendo que a escolha dependerá principalmente da disponibilidade de cada um na região de criação.

Em regiões de tráfego intenso no interior do galpão avícola, como nas proximidades dos comedouros e bebedouros, ou nas vizinhanças dos exaustores, geralmente há a formação de uma camada compacta de cama que freqüentemente possui alta umidade, e é chamada de torta (Miles et al., 2008). Quando a cama e a torta são retiradas dos galpões avícolas, podem ser utilizadas como fontes de fertilizantes para as culturas agrícolas, ao passo em que a degradação dos compostos nitrogenados presentes nas excretas em $\mathrm{NH}_{3}$ constitui um fator de empobrecimento do potencial fertilizante da cama, podendo ser detrimental para o meio ambiente (Hansen et al., 1989).

O potencial fertilizante da cama de frango de corte depende de vários fatores, dentre eles está o número de reutilizações. Pode-se observar na Tabela 2 a composição química de camas de frangos de corte com casca de café depois de utilizada de 1 a 4 vezes, cujas amostras foram retiradas de uma mesma granja. 
Tabela 2. Parâmetros e composição química da cama de frango com casca de café em função do número de reutilizações da cama.

\begin{tabular}{|c|c|c|c|c|}
\hline \multirow{2}{*}{ Nutrientes/parâmetros } & \multicolumn{4}{|c|}{ Número de criadas } \\
\hline & 1 & 2 & 3 & 4 \\
\hline Nitrogênio (\%) & 4,7 & 2,8 & 3,1 & 6,7 \\
\hline Fósforo (\%) & 2,1 & 2,8 & 4,3 & 2,4 \\
\hline Potássio (\%) & 4,4 & 4,5 & 5,3 & 3,6 \\
\hline Cálcio (\%) & 1,6 & 1,7 & 1,8 & 1,7 \\
\hline Magnésio (\%) & 0,4 & 0,5 & 0,5 & 0,5 \\
\hline Sódio (\%) & 0,5 & 0,6 & 0,6 & 0,5 \\
\hline Ferro (\%) & 0,1 & 0,1 & 0,1 & 0,2 \\
\hline Cobre (ppm) & 400 & 500 & 600 & $<0,01$ \\
\hline Manganês (ppm) & 300 & 400 & 400 & 400 \\
\hline Zinco (ppm) & 300 & 400 & 400 & 400 \\
\hline Enxofre (\%) & 0,5 & 0,5 & 0,6 & 0,6 \\
\hline Alumínio (\%) & 0,2 & 0,1 & 0,2 & 0,3 \\
\hline Cinzas (\%) & 19,4 & 16,7 & 17,9 & 21,3 \\
\hline Umidade (\%) & 29,3 & 27,8 & 29,2 & 21,3 \\
\hline Matéria orgânica (\%) & 80,6 & 83,3 & 82,1 & 78,7 \\
\hline Boro (ppm) & 10 & 11,4 & 12,3 & 11,1 \\
\hline Cobalto (ppm) & 33 & 22 & 17 & - \\
\hline Molibdênio (ppm) & 83 & 167 & 83 & - \\
\hline $\mathrm{pH}$ & 8,9 & 8,9 & 8,9 & - \\
\hline Densidade (g/cm3) & 0,33 & 0,33 & 0,39 & - \\
\hline
\end{tabular}

Fonte: Beneditti et al., 2009; Zapata, 2011.

Tem-se observado que o conteúdo de $\mathrm{N}$ na cama de frangos tende a aumentar com sua reutilização em sucessivos lotes, podendo atingir o conteúdo máximo após o quarto ou quinto lote, sendo que a quantidade de substrato novo adicionado entre lotes também irá influenciar o conteúdo final de nutrientes do material (Zapata, 2011). 
Zapata (2011) ao avaliar quimicamente o teor de $\mathrm{N}$ amoniacal $\left(\mathrm{N}-\mathrm{NH}_{3}\right)$ de amostras de cama em vários ciclos, observou que há uma correlação positiva entre estas duas variáveis, sugerindo um aumento do potencial fertilizante e também poluente da cama com o aumento do número de reutilizações.

Com relação ao teor de $\mathrm{N}$ na cama de aves para diferentes idades das aves, o acúmulo de esterco oriundo do lote em crescimento faz com que o teor de $\mathrm{N}$ na cama também aumente com o tempo, em um mesmo ciclo. Miles et al. (2008) observou que os teores de $\mathrm{N}$ total e amônio $\left(\mathrm{NH}_{4}{ }^{+}\right)$em cama de frangos de corte aumentam de 20.946 e $4.370 \mathrm{mg} \mathrm{kg}^{-1}$, para aves aos 2 - $\mathrm{d}$ de idade para 23.694 e $6.917 \mathrm{mg} \mathrm{kg}^{-1}$, para aves aos $45-\mathrm{d}$, respectivamente. Miles et al. (2008) também observou que como uma conseqüência da maior quantidade de $\mathrm{NH}_{4}{ }^{+}$na cama com a idade, também as perdas de $\mathrm{NH}_{3}$ por volatilização para o ar subjacente aumentam com a idade das aves.

\section{$\underline{1.3 \text { Animais vivos e mortos }}$}

Do total de $\mathrm{N}$ presente na ração animal, o percentual que ficará retido nas aves varia com a idade (com a variação do índice de conversão alimentar) e com o tipo de manejo. Patterson et al. (1998) observou que em granjas de frangos de corte nos Estados Unidos, aproximadamente $51 \%$ (Tabela 3) de todo o $\mathrm{N}$ que entrou no galpão na forma de ração, passou a fazer parte da constituição da carcaça das aves. Malone et al. (1987), citado por Blake (2004) observou que a carcaça fresca de um frango de corte contém aproximadamente $51,8 \%$ de proteína.

Yang et al. (2000) ao realizar o balanço de $\mathrm{N}$ para galpões de galinhas poedeiras de Iowa (E.U.A.), utilizou o valor sugerido por Haque et al. (1991) de $58 \%$ como teor médio de proteína bruta na carcaça das aves. Estes resultados concordam razoavelmente com o índice de conversão alimentar médio de linhagens comerciais de 2,0, implicando que cerca de $50 \%$ da ração oferecida às aves não será assimilada por elas. 
Tabela 3. Médias dos valores do fracionamento dos nutrientes da ração (\%) em lotes comerciais de frangos de corte nos Estados Unidos.

\begin{tabular}{|c|c|c|c|c|c|}
\hline Nutriente & Ração & Cama & Carcaça & Soma $^{A}$ & Resíduo $^{B}$ \\
\hline \multicolumn{6}{|l|}{ Compania A } \\
\hline $\begin{array}{c}\text { Nitrogênio } \\
\text { total }\end{array}$ & 100 & 30,15 & 51,23 & 81,38 & 18,62 \\
\hline Fósforo & 100 & 61,28 & 34,83 & 96,11 & 3,89 \\
\hline Potássio & 100 & 87,02 & 20,50 & 107,52 & $-7,52$ \\
\hline \multicolumn{6}{|l|}{ Compania B } \\
\hline $\begin{array}{c}\text { Nitrogênio } \\
\text { total }\end{array}$ & 100 & 30,98 & 50,94 & 81,92 & 18,08 \\
\hline Fósforo & 100 & 53,52 & 35,11 & 88,64 & 11,36 \\
\hline Potássio & 100 & 73,05 & 21,36 & 94,41 & 5,59 \\
\hline \multicolumn{6}{|l|}{ Compania C } \\
\hline $\begin{array}{c}\text { Nitrogênio } \\
\text { total }\end{array}$ & 100 & 30,56 & 51,08 & 81,64 & 18,36 \\
\hline Fósforo & 100 & 57,40 & 34,97 & 92,37 & 7,63 \\
\hline Potássio & 100 & 80,04 & 20,93 & 100,97 & $-0,97$ \\
\hline \multicolumn{6}{|c|}{$\begin{array}{l}\text { B A Percentagem residual de cada nutriente é calculada da diferença } \\
\text { entre os nutrientes da ração que entram no galpão e a soma dos } \\
\text { nutrientes da cama e da carcaça que saem do galpão: Resíduo = } \\
\text { Ração - (Cama + Carcaça) }\end{array}$} \\
\hline
\end{tabular}

Fonte: Patterson et al., 1998.

Um fato comum com o qual os produtores avícolas têm que lidar no diaa-dia é a taxa de mortalidade (TM) das aves, que chega a alcançar valores expressivos, especialmente para sistemas de criação com alta densidade. Heier et al. (2002), ao discorrer sobre as causas comuns de mortalidade de frangos de corte na Noruega, reportou que a TM depende de fatores como idade das 
aves, tamanho do lote, densidade de alojamento, tipos de sistema de ventilação, sistema de bebedouro e isolamento do piso. Após a análise estatística de 1664 lotes de frangos de corte em 132 diferentes centros de integração na Noruega, Heier et al. (2002) concluíram que na primeira semana de vida das aves, a mortalidade acumulada média foi de 1,54\%, diminuindo para $0,48 \%$ para o restante do período de crescimento. Por outro lado, Blake (2004) observou que para um lote de 50.000 frangos de corte no estado do Alabama (E.U.A.) a mortalidade acumulada ao final de $49 \mathrm{~d}$ foi de $4,9 \%$.

Com base no exposto, nota-se que a mortalidade constitui uma importante causa de perda de $\mathrm{N}$ de um sistema de produção animal, e deve ser cuidadosamente contabilizada nos cálculos de balanço de $\mathrm{N}$ para galpões avícolas.

\section{Os principais processos que envolvem o $\mathrm{N}$ no interior de um galpão avícola}

\subsection{Oxidação de aminoácidos e produção de ácido úrico}

Os aminoácidos derivados das proteínas da ração ou da degradação das proteínas intracelulares são a ultima classe de biomoléculas, cuja oxidação faz uma contribuição significativa para a geração de energia metabólica. Um fator muito importante distingue a degradação dos aminoácidos dos processos metabólicos de degradação de lipídios e carboidratos: todos os aminoácidos contêm um grupo amino. Sendo assim, cada via de degradação passa por um passo-chave no qual o grupo a-amino é separado do esqueleto carbônico e desviado para uma via especializada que culminará no metabolismo do grupamento amino (Nelson e Cox, 2009).

Uma vez ingeridos pelo animal, os aminoácidos (ou proteínas, após digeridas pelo trato gastro-intestinal) são adsorvidos e encaminhados para as células, onde podem servir a duas finalidades distintas: (a) síntese de outros aminoácidos e subseqüentemente de proteínas utilizáveis pelo organismo para a construção de tecidos diversos e em especial tecidos musculares, e (b) degradação catabólica para a liberação de energia para ser utilizada para a 
síntese de biomoléculas. De acordo com Nelson e Cox (2009), o caminho (b) ocorre naturalmente nos animais, porém em uma intensidade maior apenas em casos de jejum severo, onde há a escassez de outras fontes prioritárias de energia (carboidratos e lipídeos). Do ponto de vista da produção animal, e em especial da criação de frangos de corte, as dietas formuladas são ricas em energia metabolizável o suficiente para fazer com que o caminho (b) ocorra com a menor freqüência possível.

Os aminoácidos derivados das proteínas dos alimentos são a fonte da maioria dos grupos aminos, sendo a maior parte metabolizada no fígado. Parte da $\mathrm{NH}_{3}$ assim gerada é reciclada e empregada em uma grande variedade de processos biossintéticos, sendo o excesso excretado diretamente ou convertido em uréia ou ácido úrico. $\mathrm{A} \mathrm{NH}_{3}$ em excesso gerada em outros tecidos é transportada para o fígado para a conversão na forma apropriada de excreção (Nelson e Cox, 2009).

Por serem animais uricotélicos, as aves produzem o ácido úrico como excremento do $\mathrm{N}$ excedente que não foi utilizado na síntese de moléculas bioquímicas (Nelson e Cox, 2009). Os animais domésticos em geral, e em especial os frangos de corte são alimentados com dietas contendo altos níveis de proteínas, parte das quais será excretada na forma não digerida, e a parcela que foi digerida resultará na liberação de ácido úrico. Tanto o ácido úrico quanto as proteínas não digeridas presentes no esterco dos frangos de corte, entrarão em contato com a cama após a defecação e estarão susceptíveis a uma série de outros processos bioquímicos que culminarão na produção de $\mathrm{NH}_{4}{ }^{+}$, e finalmente $\mathrm{NH}_{3}(\mathrm{~g})$.

\subsection{Mineralização do $\mathrm{N}$ presente nos excrementos das aves}

Uma vez em contato com as condições ambientais favoráveis, a cama entrará em processo de degradação bacteriológica, resultando na formação de substâncias simples; o processo de degradação se prolongará enquanto houver matéria prima, sendo os produtos inorgânicos finais volatilizados ou 
assimilados por outros organismos, como as plantas, na eventualidade da cama ser utilizada como adubo.

De todos os processos que constituem o ciclo biogeoquímico do $\mathrm{N}$ na natureza, o processo de maior importância que ocorre ainda no interior do galpão é a mineralização. De acordo com Manzoni e Porporato (2009), o processo de mineralização é definido como sendo uma série complexa de reações químicas que é governada principalmente por meios biológicos, na qual um substrato orgânico é convertido em biomassa viva e resíduos minerais.

\section{$\underline{2.3 \text { Volatilização }}$}

Volatilização é o nome que se dá ao processo físico de passagem de uma substância do estado líquido para o estado gasoso, com conseqüente transporte convectivo da substância gasosa. A taxa de volatilização da $\mathrm{NH}_{3}$ da cama de aves depende diretamente do teor de $\mathrm{NH}_{3}$ dissolvido na solução que envolve as partículas da cama. Por outro lado, a disponibilidade de $\mathrm{NH}_{3}$ na solução da cama depende de fatores como $\mathrm{pH}$, teor de umidade, temperatura e concentração de $\mathrm{NH}_{4}{ }^{+}$, que regem a conversão entre $\mathrm{NH}_{4}{ }^{+}$(com baixo grau de volatilização) em $\mathrm{NH}_{3}$ (altamente volatilizável) (Zapata, 2011).

Várias teorias têm sido desenvolvidas para explicar o processo de volatilização da $\mathrm{NH}_{3}$ dos excrementos animais. Sendo que duas das quais têm sido as mais utilizadas em estudos recentes.

a)Teoria dos dois filmes

Esta teoria tem sido utilizada na modelagem de sistemas contendo solo (Jayaweera, 1990; Wang, 2010), e possui duas pressuposições importantes. A primeira é que a taxa de transferência entre filmes é regida pelas taxas de difusão através de cada uma das interfaces. A outra pressuposição implica que não há nenhuma outra resistência durante a transferência da $\mathrm{NH}_{3}$ líquida ou gasosa através das interfaces (Weltry, 1984; Wang, 2010). Podem-se observar na Figura 3 o esquema e os parâmetros utilizados na teoria dos dois filmes. 
MENDES, L.B. et al. O ciclo do nitrogênio na criação de frangos de corte e suas perdas na forma de amônia volátil: uma revisão. PUBVET, Londrina, V. 6, N. 20, Ed. 207, Art. 1383, 2012.

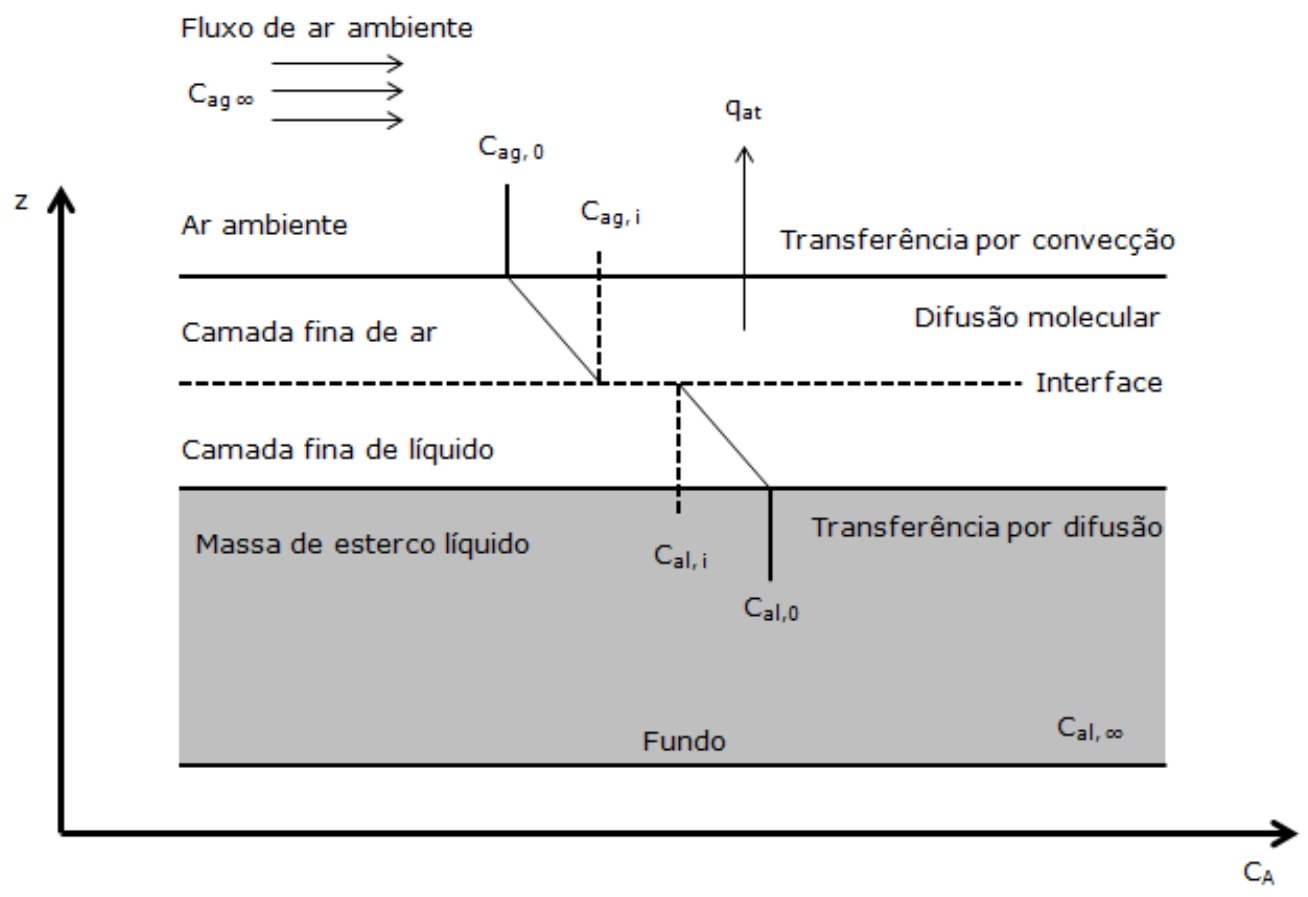

Figura 3. Ilustração da teoria dos dois filmes para explicar a volatilização da $\mathrm{NH}_{3}$ (Adaptado: $\mathrm{Ni}, 1999$ ).

A espessura dos filmes de líquido e gás é muito pequena em relação à espessura da camada de esterco ou cama. Três passos envolvem o processo de transferência da $\mathrm{NH}_{3}$, os quais são: (a) difusão da $\mathrm{NH}_{3}$ de dentro da massa de esterco ou cama para a superfície; (b) transferência da $\mathrm{NH}_{3}(\mathrm{I})$ através da camada líquida superficial e (c) transferência convectiva da $\mathrm{NH}_{3}(\mathrm{~g})$ através do filme constituído por gás e daí para o ar ambiente $(\mathrm{Ni}, 1999)$.

b) Teoria da camada limite

De acordo com esta teoria, a volatilização da $\mathrm{NH}_{3}$ ocorre em dois passos: (a) difusão através da camada líquida que envolve as partículas de esterco ou cama, e (b) transferência convectiva da superfície líquida através da camada limite e daí para o ambiente (Figura 4) (Ni, 1999). 
MENDES, L.B. et al. O ciclo do nitrogênio na criação de frangos de corte e suas perdas na forma de amônia volátil: uma revisão. PUBVET, Londrina, V. 6, N. 20, Ed. 207, Art. 1383, 2012.

Fluxo de ar ambiente

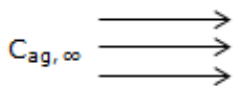

Transferência por convecção

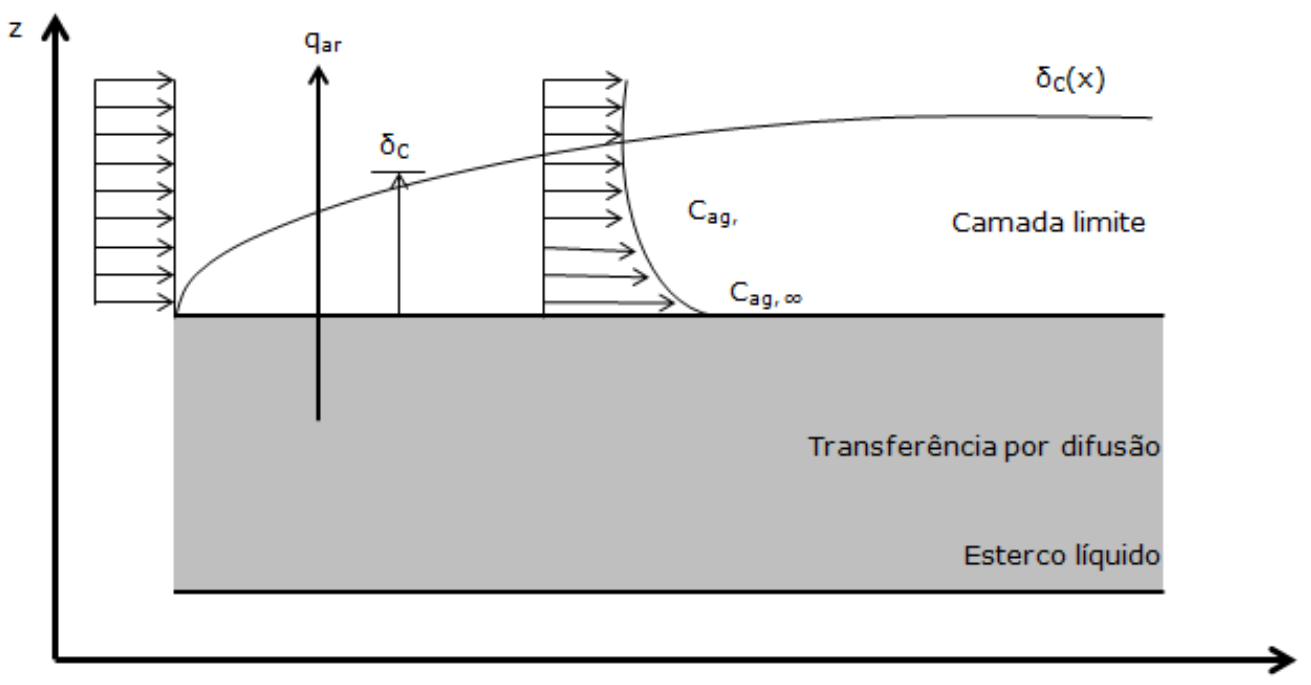

Figura 4. Ilustração da teoria da camada limite para explicar a volatilização da $\mathrm{NH}_{3}$ (Adaptado: $\mathrm{Ni}, 1999$ ).

A lei de Fick (Equação 2) foi utilizada por Ni (1999) para descrever matematicamente a transferência de $\mathrm{NH}_{3}$ da superfície líquida que envolve as partículas de esterco ou cama.

$j=D_{g} \frac{C_{g, 0}-C_{g}}{\delta_{g}}=k_{g}\left(C_{g, 0}-C_{g}\right) \approx k_{g} C_{g, 0}$

Em que $\mathrm{j}\left(\mathrm{kg} \mathrm{m}^{-2} \mathrm{~s}^{-1}\right)$ é o fluxo mássico de $\mathrm{NH}_{3} ; \mathrm{D}_{\mathrm{g}}$ é o coeficiente de difusão da $\mathrm{NH}_{3}$ no ar; $\mathrm{C}_{g}$ é a concentração de $\mathrm{NH}_{3}\left(\mathrm{~kg} \mathrm{~m}^{-3}\right)$ acima da camada limite; $\mathrm{C}_{g, 0}$ é a concentração de $\mathrm{NH}_{3}$ na camada limite subjacente à superfície da camada líquida que envolve a partícula de esterco ou cama; $\delta_{g}$ é a espessura da camada limite laminar $(\mathrm{m})$; e $\mathrm{k}_{\mathrm{g}}$ é o coeficiente convectivo de transferência de $\mathrm{NH}_{3}$ no ar acima da camada limite $\left(\mathrm{m} \mathrm{s}^{-1}\right)$. 


\subsection{Relação entre teor de $\mathrm{N}$ na cama e a emissão de $\mathrm{NH}_{3}$}

A fonte principal da $\mathrm{NH}_{3}$ emitida para a atmosfera num galpão de frangos de corte é a cama, e a intensidade da volatilização deste gás depende de sua disponibilidade e das propriedades da cama. De acordo com Li (2006) os fatores mais importantes na volatilização da $\mathrm{NH}_{3}$ são $\mathrm{pH}$, umidade relativa e temperatura da cama. Na medida em que a disponibilidade de excretas das aves na cama aumenta, aumenta também a população microbiana responsável pela mineralização da matéria orgânica e transformação do ácido úrico presentes na cama e aumento do seu teor de $\mathrm{NH}_{4}{ }^{+}$.

Gates et al. (2008) reportou que a taxa de emissão de $\mathrm{NH}_{3}\left(\mathrm{TE}_{\mathrm{NH} 3}\right)$ para frangos de corte pode ser escrita como uma função da idade da ave, sendo a relação linear (Equações 2 e 3).

$T E=0,031( \pm 1,1) x$

idade da ave (d), se a cama for reutilizada

$x=0, \quad$ se a cama for nova e a idade da ave $<7$

idade da ave -6 , se a cama for nova e a idade da ave $\geq 7$

Em que:

$\mathrm{TE}_{\mathrm{NH} 3}=$ taxa de emissão de $\mathrm{NH}_{3}\left(\mathrm{~g} \mathrm{~d}^{-1}\right.$ ave $\left.{ }^{-1}\right)$;

Uma relação similar entre a taxa de emissão de $\mathrm{NH}_{3}$ e a idade das aves têm sido reportado por Mendes (2010), Saraz (2010), Roumeliotis et al. (2010)(a) e (b), Wheeler et al. (2006), Guiziou e Béline (2005).

$\mathrm{O}$ percentual de $\mathrm{N}$ total que entra num galpão avícola e que é perdido para a atmosfera através da volatilização da $\mathrm{NH}_{3}$ pode ser determinado de forma direta ou indireta. Um método direto para estimar o percentual de $\mathrm{NH}_{3}$ perdido para a atmosfera consiste no monitoramento (contínuo ou semicontínuo) da taxa de emissão de $\mathrm{NH}_{3}$ durante todo o ciclo de criação dos frangos de corte. 
Para o método indireto, também chamado de balanço de $\mathrm{N}$, a perda de $\mathrm{N}$ na forma de $\mathrm{NH}_{3}$ é estimada a partir da diferença algébrica entre os conteúdos de $\mathrm{N}$ da ração e da cama e das aves.

A técnica do balanço de massa tem sido considerada pelo National Research Council (2003) como sendo o melhor método para estimar emissões de $\mathrm{NH}_{3}$. No modelo do balanço de massa, os meios de entrada e saída de $\mathrm{N}$ do galpão avícola são quantificados, e a diferença entre o total de entrada e saída é tida como sendo $\mathrm{N}$ volatilizado. Esta técnica, porém, não distingue as perdas de $\mathrm{N}$ entre $\mathrm{NH}_{3}$ e outros gases como o gás $\mathrm{N}\left(\mathrm{N}_{2}\right)$ ou NOx; outra desvantagem é que ele não caracteriza os ciclos diários ou os valores das concentrações máximas dos gases. Contudo, ela pode fornecer uma estimativa bastante precisa dos valores máximos das emissões de $\mathrm{NH}_{3}$ ao longo de períodos prolongados de tempo (vários dias ou um ciclo completo) (Keener e Zhao, 2006; Liu et al., 2006).

Guiziou e Béline (2005) realizaram o balanço de N para um galpão de frango de corte, e monitoraram a emissão de $\mathrm{NH}_{3}$ para determinar as perdas de $\mathrm{N}$ para a atmosfera. Os percentuais de $\mathrm{N}$ retidos na carcaça das aves, na cama e perdidos na forma de gases para a atmosfera e em especial na forma de $\mathrm{NH}_{3}$ podem ser observados na Figura 5.

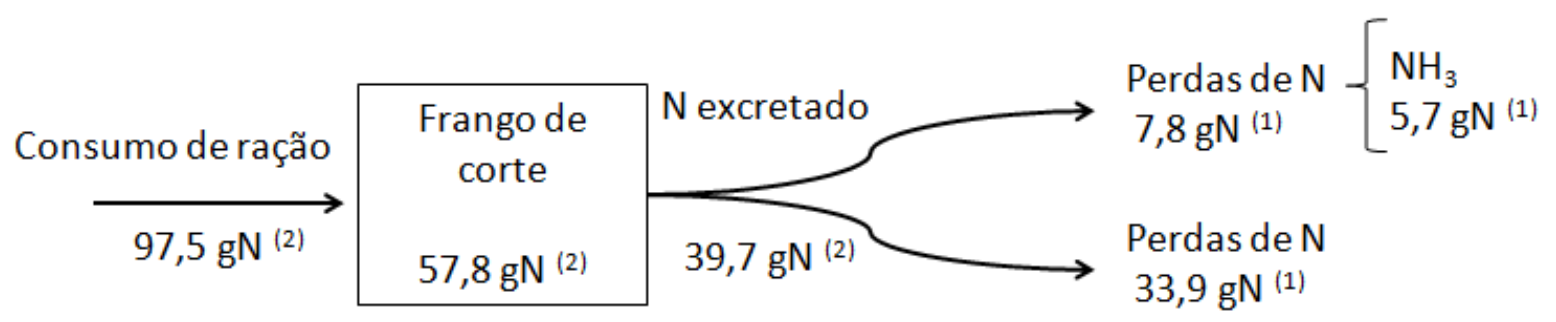

Figura 5. Balanço de $\mathrm{N}$ estimado para um ciclo completo de frangos de corte em um galpão. Os números subscritos significam: (1) valores medidos e (2) valores calculados (Adaptado de Guiziou e Béline, 2005). 
Diversos métodos de balanço de $\mathrm{N}$ têm sido utilizados por vários autores como uma forma de estimar perdas de $\mathrm{N}$ amoniacal $\left(\mathrm{N}-\mathrm{NH}_{3}\right)$ a partir de sistemas comerciais de produção avícola (Liang et al., 2004; Keener and Zhao, 2008; Yang et al., 2000; Powell et al., 2008; Blanes-vidal et al., 2007). Para exemplificar, Keener e Zhao (2008) estimaram o total de $\mathrm{NH}_{3}-\mathrm{N}$ emitido para atmosfera em dois diferentes tipos de alojamentos para poedeiras utilizando a técnica do balanço de massa. Os resultados do balanço para dois ensaios realizados no mesmo alojamento podem ser observados na Tabela 4.

Tabela 4. Balanço de $\mathrm{N}$ para dois tipos diferentes de alojamento para poedeiras, com ênfase para as perdas de $\mathrm{N}$ na forma de $\mathrm{NH}_{3}-\mathrm{N}$.

\begin{tabular}{|c|c|c|c|c|}
\hline $\begin{array}{l}\text { Tipo de } \\
\text { sistema }\end{array}$ & $\mathrm{N}_{\text {ração }}(\%)$ & $\mathrm{N}_{\text {ovos }}(\%)$ & $\mathrm{N}_{\text {esterco }}(\%)$ & $\begin{array}{c}\mathrm{NH}_{3}-\mathrm{N} \text { perdido } \\
(\%)\end{array}$ \\
\hline \multicolumn{5}{|l|}{ Ensaio 1} \\
\hline DP & 100,0 & 16,2 & 26,5 & 57,5 \\
\hline $\mathrm{MB}+\mathrm{CB}$ & 100,0 & 16,2 & 68,7 & 15,2 \\
\hline \multicolumn{5}{|l|}{ Ensaio 2} \\
\hline DP & 100,0 & 16,2 & 38,0 & 45,8 \\
\hline $\mathrm{MB}+\mathrm{CB}$ & 100,0 & 16,2 & 67,3 & 16,5 \\
\hline $\begin{array}{l}\text { DP - sistema } \\
\text { aves alojada } \\
\text { armazenado } \\
\text { MB - sistem } \\
\text { baterias de } \\
\text { remoção do } \\
\text { CB - área or } \\
\text { e submetido }\end{array}$ & $\begin{array}{l}\text { ma alojamer } \\
\text { paviolas no } \\
\text { para poedeira } \\
\text { las possuen } \\
\text { erco para a } \\
\text { o esterco or } \\
\text { ompostager }\end{array}$ & $\begin{array}{l}\text { to de poede } \\
\text { pavimento } \\
\text { nferior. } \\
\text { s com um ú } \\
\text { correias tr } \\
\text { rea de arm } \\
\text { iundo do sis } \\
\text {. }\end{array}$ & $\begin{array}{l}\text { nico pavimen } \\
\text { ansportadora } \\
\text { azenamento; } \\
\text { tema tipo ME }\end{array}$ & $\begin{array}{l}\text { o, no qual as } \\
\text { para a coleta e } \\
\text { é armazenado }\end{array}$ \\
\hline
\end{tabular}

Adaptado de Keener e Zhao, 2008. 
Keener e Zhao (2008) concluíram que o método do balanço de $\mathrm{N}$ proposto para estimar as perdas de $\mathrm{NH}_{3}-\mathrm{N}$ possui baixo custo, pode ser facilmente implementado, é confiável e possui acurácea razoável quando comparado com o monitoramento direto da emissão de $\mathrm{NH}_{3}$.

\section{Considerações finais sobre o balanço de nitrogênio para um galpão avícola}

Em suma, o balanço de $\mathrm{N}$ para um galpão avícola (volume de controle, VC) pode envolver todos os processos que descrevem o ciclo do $\mathrm{N}$ na natureza, sendo que os mais importantes são a síntese de ácido úrico no organismo das aves e posterior excreção; a mineralização dos compostos orgânicos nitrogenados e conseqüente formação de substâncias como $\mathrm{NH}_{4}{ }^{+}, \mathrm{NH}_{3}, \mathrm{NO}_{\text {x }}$, dentre outras; e volatilização dos compostos inorgânicos nitrogenados presentes na cama.

A ração oferecida às aves é considerada a principal fonte de $\mathrm{N}$ para o VC, enquanto que as principais vias de saída de $\mathrm{N}$ são a mortalidade e as perdas gasosas por substâncias nitrogenadas voláteis.

A relação entre o teor de nitrogênio da cama e do organismo das aves é regida pelo índice de conversão alimentar (CA), o qual aumenta com o envelhecimento das aves, chegando a aproximadamente 2,0 na idade de abate, significando que aos $45 \mathrm{~d}$ de idade, $50 \%$ do nitrogênio que entra no VC é absorvido pelas aves, ao passo que $50 \%$ é excretado na cama.

A quantidade da $\mathrm{NH}_{3}$ emitida da cama avícola possui relação direta com o teor de $\mathrm{N}$ total $\left(\mathrm{N}_{\text {total }}\right)$ da mesma, sendo que o $\mathrm{N}_{\text {total }}$ aumenta com a idade das aves e com o número de reutilizações, sendo que o teor máximo é alcançado entre a $4^{\mathrm{a}}$ e $5^{\mathrm{a}}$ criada.

A quantidade de $\mathrm{NH}_{3}$ perdida do VC através de fluxos gasosos pode ser aferida diretamente pelo monitoramento da taxa de emissão e indiretamente pelo balaço de $\mathrm{N}$ para o $\mathrm{VC}$ considerado. 
MENDES, L.B. et al. O ciclo do nitrogênio na criação de frangos de corte e suas perdas na forma de amônia volátil: uma revisão. PUBVET, Londrina, V. 6, N. 20, Ed. 207, Art. 1383, 2012.

O percentual de $\mathrm{N}$ que entra no $\mathrm{VC}$ perdido com a volatilização de gases nitrogenados (sobretudo $\mathrm{NH}_{3}$ ) varia de acordo com o manejo, idade das aves, tipo de ração, condições da cama, etc., mas pode-se dizer que corresponde, em média, a $35 \%$ de todo o $\mathrm{N}$ que entra no galpão.

\section{Referências}

Benedetti, M.P.; Fugiwara, A.T.; Factori, M.A.; Costa, C.; Meirelles, P.R.L. Adubação com cama de frango em pastagem. Águas de Lindóia: ZOOTEC, 2009.

Blake, J. P. 2004. Methods and technologies for handling mortality losses. World's Poultry Science Journal, v.60, p.489-499. DOI:10.1079/WPS200432.

Calvet, S.; Cambra-López, M.; Blanes-Vidal, V.; Estellés, F.; Torres, A. G. 2010. Ventilation rates in mechanically-ventilated commercial poultry buildings in Southern Europe: Measurement system development and uncertainty analysis. Biosystems Engineering 106 (2010) 423-432.

Cobb-Vantress, 2009. Suplemento de crescimento e nutrição para frangos de corte, Cobb500 ${ }^{\mathrm{TM}}$. Disponível em: WWW.cobb-vantress.com, acessado em 23/09/2011.

Erisman, J. W.; Bleeker, A.; Galloway, J.; Sutton, M. S. 2007. Reduced nitrogen in ecology and the environment. A literature review. Environmental Pollution, 150 (2007) 140-149.

Follet, R. F.; Hatfield, J. L. 2009. Nitrogen in the environment: Sources, problems and management, 5a Edição. Elsevier, San Diego, CA, USA, 525 p.

Gates, R. S.; Casey, K. D.; Wheeler, E. F.; Xin, H.; Pescatore, A. J. 2008. U. S. broiler housing ammonia emissions inventory. Atmospheric Environment 42 (2008) 3342-3350.

Green, A. R.; Wesley, I.; Trampel, D. W.; Xin, H. 2009. Air quality and bird health status in three types of commercial egg layer houses. J. Appl. Poult. Res. 18:605-621. DOI:10.3382/japr.2007-00086.

Guiziou, F.; Béline, F. 2005. In situ measurement of ammonia and greenhouse gas emissions from broiler house in France. Bioresource Technology 96 (2005) 203-207.

Hansen, R. C., H. M. Keener, and H. A. J. Hoitink. 1989. Poultry manure composting: An exploratory study. Transactions of the ASAE 32(6): 2151-2158.

Haque, A. K. M. A., J. J. Lyons, and J. M. Vandepopuliere. 1991. Extrusion processing of broiler starter diets containing ground whole hens, poultry by-product meal, feather meal, or ground feathers. Poultry Sci. 70(2): 234-240.

Hartung, J. 1990. Influence of housing and livestock on ammonia release from buildings. In Odour and Ammonia Emissions from Livestock Farming, eds. V. C. Nielsen, J. H. Voorburg, and P. L'Hermitte. The Netherlands: Elsevier Applied Science.

Heier, B. T.; Hogasen, H. R.; Jarp, J. 2002. Factors associated with mortality on Norwegian broiler flocks. Preventive Veterinary Medicine 53 (2002) 147-158. 
Jayaweera, G.R., D.S. Mikkelson. 1990. Ammonia volatilization from flooded soil systems: A computer model. I. Theoretical aspects. Soil Science Society of America Journal 54: 14471455.

Keener, H. M. and Zhao, L. 2006. Predicting NH3 Emissions from Manure N for Livestock Facilities and Storages: A Modified Mass Balance Approach. Proceedings of the Workshop on Agricultural Air Quality: State of the Science. June 5-8, Bolger Center, Potomac, MD, USA.

Keener, H. M.; Zhao, L. 2008. A modified mass balance method for predicting $\mathrm{NH}_{3}$ emissions from manure $\mathrm{N}$ for livestock and storage facilities. Biosystems Engineering 99 (2008) 81-87.

Krupa, S. V. 2003. Effects of atmospheric ammonia (NH3) on terrestrial vegetation: a review. Environmental Pollution, 124 (2003) 179-221.

Li, Hong. 2006. Ammonia emissions from manure belt laying hen houses and manure storage. PhD Dissertation. Ames, IA.: Iowa State University, Department of Agricultural and Biosystems Engineering.

Liang et al., 2004; Keener and Zhao, 2008; Yang et al., 2000; Powell et al., 2008; Blanes-vidal et al., 2007

Liang, Y., H. Xin, and E.F. Wheeler. 2004. Ammonia emission from U.S. laying hen houses in Iowa and Pennsylvania. Transactions of the ASAE 48(5): 1927-1941.

Liu, Z.; Wang, L.; Beasley, D. B. 2006. A review of Emission Models of Ammonia Released from Broiler Houses. ASABE Technical Paper 06-4101, St. Portland, OR: ASABE.

Malone, G.W., Saylor, W.W., Ariza, M.G., Lomax, K.M. And Kaifer, C.R. 1987. Acid preservation and utilization of poultry carcasses resulting from mortality losses. Pages 13-16 in: Progress Through Research and Extension 1987, Report 11. University of Delaware College of Agricultural Sciences, Newark, DE, USA.

Manzoni, S.; Porporato, A. 2009. Soil carbon and nitrogen mineralization: Theory and models across scales. Soil Biology and Biochemistry 41 (2009) 1355-1379.

Mendes, L. B. 2010. Ammonia emissions, feeding and defecation dynamics of W-36 pullets and laying hens as affected by stocking density and manure accumulation time. Master's Thesis. Ames, IA.: Iowa State University, Department of Agricultural and Biosystems Engineering.

Miles, D. M.; Branton, S. L.; Lott, B. D. 2004. Atmospheric ammonia is detrimental to the performance of modern commercial broilers. Poultry Science 83:1650-1654.

Miles, D.M., D.E. Rowe and P.R.Owens. 2008. Winter broiler litter gases and nitrogen compounds: temporal and spatial trends. Atmospheric Environment. 42: 3351-3363.

Nelson, D. L.; Cox, M. M. 2011. 2009. Lehninger: principles of biochemistry, 5th edition, W. H. Freeman and Company, New York, U. S. A.

$\mathrm{Ni}$, J. 1999. Mechanistic models of ammonia release from liquid manure: a review. Journal of Agricultural Engineering Research. 72(1): 1-17.

Patterson, P. H., and E. S. Lorenz. 1996. Manure nutrient production from commercial white leghorn hens. 1996 J. Appl. Poultry Res. 5: 260-268. 
Patterson, P. H.; Lorenz, E. S.; Weaver, W. D. 1998. Litter production and nutrients from commercial broiler chickens. J. Appl. Poult. Res. 7:247-252.

Roumeliotis, T. S.; Dixon, B. J.; van Heyst, J. 2010. Characterization of gaseous pollutant and particulate matter emission rates from a commercial broiler operation part I: Observed trends in emissions. Atmospheric Environment 44 (2010) 3770-3777.

Roumeliotis, T. S.; Dixon, B. J.; van Heyst, J. 2010. Characterization of gaseous pollutant and particulate matter emission rates from a commercial broiler operation part II: Correlated emission rates. Atmospheric Environment 44 (2010) 3778-3786.

Saraz, J. A. 2010. Determinação experimental e modelagem em CFD das taxas de emissões de amônia de camas de aviários e distribuições de concentrações, temperatura e velocidade do ar no interior de galpões avícolas. Viçosa: $122 \mathrm{f}$. Tese (Doutorado em Construções Rurais e Ambiencia). Departamento de Engenharia Agrícola, Universidade Federal de Viçosa, Viçosa, MG.

Sutton, M. A.; Erisman, J. W.; Dentener, F.; Möller, D. 2008. Ammonia in the encvironment: From ancient times to the present. A review. Environmental Pollutionn 156 (2008) 583-604.

Tinôco, I. F. F. 2002. Novas tendências em projetos para a avicultura Industrial. In: FACTA. (Org.). Novas tendências em projetos para a avicultura Industrial. Jaboticabal - SP: , v. 1, p. 1101.

Tinôco, I. F. F. 2004. A granja de frangos de corte. In: Mendes A A, Nããs IA \& Macari M (Eds.) Produção de frangos de corte. Campinas. FACTA. p. 55-84.

Wang, A. 2010. Predictation of ammonia emission from poultry layer and dairy houses using an alternative mass balance method. Tese de Mestrado. Columbus, $\mathrm{OH}$.: The Ohio State University, Department of Food, Agricultural and Biological Engineering.

Weltry, J.R., C.E. Wicks, R.E. Wilson. 1984. Fundamentals of momentum, heat, and mass transfer. 3rd ed. Wiley. New York.

Wheeler, E.F., Casey, K.D., Gates, R.S., Xin, H., Zajaczkowski, J.L., Topper, P.A., Liang, Y., Pescatore, A.J. 2006. Ammonia emissions from twelve U.S.A. broiler chicken houses. Transactions of the ASABE, v. 49, n 5, p. 1495-1512.

Xin, H.; Li, H. Burns, R. T.; Gates, R. S. Overhults, D. G. Earnerst, J. W. 2009. Use of CO2 concentration difference or $\mathrm{CO} 2$ balance to assess ventilation rate of broiler houses. Transactions of the ASABE, vol.52(4):1353-1361.

Yang, P.; Lorimor, J. C.; Xin, H. 2000. Nitrogen losses from laying hen manure in commercial high-rise layer facilities. Transactions of the ASAE, V. 43(6): 1771-1780.

Zapata, O. L. M. 2011. Caracterização e avaliação do potencial fertilizante e poluente de distintas camas de frango submetidas a reusos seqüenciais na zona da mata do estado de Minas Gerais. Viçosa: 108 f. Dissertação (Mestrado em Construções Rurais e Ambiencia). Departamento de Engenharia Agrícola, Universidade Federal de Viçosa, Viçosa, MG. 